\title{
Metodologia de Equalização Adaptativa de Canais de Comunicação via Algoritmo NLMS com o Tamanho de Passo Variável Fuzzy
}

\author{
Rodrigo Possidônio Noronha \\ Departamento de Engenharia Elétrica \\ Instituto Federal de Educação, Ciência e Tecnologia do Maranhão \\ Imperatriz, Brasil \\ rodrigo.noronha@ifma.edu.br
}

\begin{abstract}
Resumo-A equalização é uma importante aplicação da teoria de processamento de sinais para a teoria comunicação, uma vez que sua função é cancelar ruídos presentes em canais de comunicação. O desempenho de um equalizador adaptativo é influenciado pelo tamanho de passo de um algoritmo adaptativo baseado em gradiente descendente estocástico, utilizado para atualização da estimativa do vetor de pesos. Um bom desempenho na equalização adaptativa pode ser obtido através da adaptação do tamanho de passo variável. Para isso, neste artigo é proposta uma nova versão do algoritmo NLMS com o tamanho de passo variável adaptado por um Sistema de Inferência Fuzzy Mamdani, aplicado na equalização adaptativa de canais de comunicação. $O$ equalizador adaptativo foi analisado em três cenários de relação sinal-ruído com $\mathbf{S N R}=18 \mathbf{d B}, \mathbf{S N R}=24 \mathbf{d B}$ e $\mathbf{S N R}=30 \mathbf{d B}$.
\end{abstract}

Index Terms-Equalização Adaptativa, Gradiente Descendente Estocástico, NLMS, Sistemas Fuzzy, Tamanho de Passo.

\section{INTRODUÇÃO}

A demanda por uma rápida transmissão de dados em canais de comunização tem sido cada vez mais crescente. Embora a comunidade acadêmica tenha tido sucesso no desenvolvimento de novas metodologias para aumento de velocidade na transmissão de dados, a presença de ruído no canal é um fator limitante para um bom desempenho de um sistema de comunicação. Nesse sentido, a equalização de canais de comunicação é essencial para o cancelamento de ruído e garantia da integridade do sinal de entrada do sistema de comunicação [1]. Um equalizador pode ser modelado por um filtro adaptativo em que o vetor de pesos pode ser obtido através de um algoritmo adaptativo baseado, por exemplo, em gradiente descendente estocástico [2]. Diversos algoritmos adaptativos baseado em gradiente descendente estocástico têm sido utilizados para o projeto de equalizadores, com uma maior frequência utilizando os algoritmos LMS (do inglês, Least Mean Square) [3] [1] e NLMS (do inglês, Normalized Least Mean Square) [4] [5].

O presente trabalho é delimitado a discutir o algoritmo NLMS para o projeto de equalizadores, uma vez que ele apresenta uma menor sensibilidade às variações da potência do sinal de entrada e tem um bom desempenho em sinais correlacionados [6]. Uma importante parâmetro para um bom desempenho do algoritmo NLMS é o tamanho de passo. Além de influenciar na convergência e estabilidade, se o tamanho de passo é grande a velocidade de convergência do NLMS será rápida, mas o erro médio quadrático ou MSE (do inglês, Mean Squared Error) no regime estacionário será grande. Se o tamanho de passo é pequeno a velocidade de convergência do NLMS será lenta, mas o MSE no regime estacionário será pequeno [7]. A influência do tamanho de passo para o desempenho do algoritmo NLMS independe da natureza do problema [8]. Segundo [8], um bom desempenho do algoritmo NLMS pode ser obtido tornando o tamanho de passo variável ou VSS (do inglês, Variable Step Size ).

Um aspecto comum para as metodologias que propõem abordagens para ajustar o VSS é fazer com que durante os primeiros instantes de tempo o tamanho de passo seja grande e com o avanço dos instantes de tempo o tamanho de passo seja pequeno. Algumas metodologias propostas para solucionar esse problema podem ser vistas em [3], [7], [9]. As metodologias que tratam de solucionar esse problema geralmente necessitam de medidas estatísticas de alta ordem que nem sempre estão disponíveis ou não são viáveis de serem obtidas para um sistema dinâmico operando em tempo real, em que as informações dinâmicas do sistema são disponibilizadas na forma de fluxo de dados que devem ser rapidamente processadas e descartadas por limitação de memória. Além disso, o ajuste do VSS é dependente da natureza do problema, além de necessitar de informações adicionais tais como parâmetros de amortecimento, fatores de esquecimento, entre outros. Um outro grande problema é estabelecer critérios para que o VSS não ultrapasse o limite de estabilidade do algoritmo NLMS, além do incremento da complexidade de análise matemática.

Desde que o Sistema de Inferência Fuzzy Mamdani ou MFIS (do inglês, Membership Fuzzy Inference System) foi proposto, diversos problemas de difícil análise e formulação matemática têm sido solucionados por meio de uma descrição linguística implementada na forma de regras baseadas no conhecimento do especialista, como na teoria de sistemas de controle [10], em identificação de sistemas [11], em robótica [12], entre outros. O comportamento dinâmico do VSS pode ser modelado utilizando um MFIS para obtenção de um bom desempenho do algoritmo NLMS independente de medidas estatísticas 
de alta ordem. Na literatura, existem poucas metodologias propostas que tratam ajustar o VSS através de um MFIS. Em [13] foi utilizado um MFIS para o ajuste do VSS aplicado na equalização de canais, em que o tamanho de passo é ajustado somente em função do erro quadrático obtido a cada instante de tempo, não sendo levado em consideração nenhuma informação sobre a quantidade de instantes de tempo necessários para a convergência do vetor de pesos de um filtro adaptativo. Uma vez que se espera que ao fim dos instantes de tempo ocorra a convergência do vetor de pesos do filtro adaptativo, a quantidade de instantes de tempo é um parâmetro importante para a otimização do funcional de custo descrito pelo gradiente estocástico.

Este artigo tem como objetivo propor a equalização adaptativa de canais de comunicação lineares e invariantes no tempo através de uma nova versão do algoritmo NLMS com o VSS adaptado por um MFIS, nomeado FVSS-NLMS (do inglês, Fuzzy Variable Step Size - Normalized Least Mean Square). No algoritmo FVSS-NLMS, o VSS é adaptado em função do erro quadrático e do instante de tempo normalizado pelo método Min-Max. Através de uma descrição linguística implementada em uma base de regras fuzzy, é possível obter uma melhoria no desempenho do equalizador, quando comparado à utilização do tamanho de passo fixo. Dessa forma, a contribuição proposta nesse trabalho é clara e justificada. Esse artigo é organizado como segue: na Seção II, é apresentada a declaração do problema de equalização; na Seção III, é apresentado o algoritmo FVSS-NLMS; na Seção IV, são apresentados os resultados obtidos na equalização de um canal de comunicação na presença de um ruído com as relações sinal-ruído iguais a $\mathrm{SNR}=18 \mathrm{~dB}, \mathrm{SNR}=24 \mathrm{~dB}$ e $\mathrm{SNR}=30 \mathrm{~dB}$.

\section{DeclaraçÃo do Problema de EqualizaÇÃo AdAPTATIVA DE CANAIS DE COMUNICAÇÃo}

O objetivo de um equalizador é reconstruir o sinal transmitido por um canal de comunicação. De acordo com [14], um equalizador é definido como um filtro projetado para contrabalançar as incertezas contidas em um determinado canal de comunicação que modificam o sinal de mensagem enviado. Seja o sinal de entrada do equalizador dado por:

$$
x(k)=\sum_{i=0}^{M-1} h(i) a(k-i)+v(k),
$$

em que $h(i)$ com $i=0,1,2, \ldots, M-1$ é a resposta impulsiva do canal linear e invariante no tempo, $v(k)$ é o sinal de ruído gaussiano aditivo com variância $\sigma_{v}^{2}, a(k)$ é o sinal de entrada codificado descrito por uma sequência binária $\{ \pm 1\}, k$ é o instante de tempo e $M$ é a quantidade de pesos do equalizador. O sinal de saída do equalizador é dado por:

$$
\hat{d}(k)=\boldsymbol{\Theta}^{T}(k) \mathcal{X}(k)=\mathcal{X}^{T}(k) \Theta(k),
$$

em que $\mathcal{X}(k)=[x(0) x(1) \ldots x(M-1)] \in \mathbb{R}^{M \times 1}$ é o vetor de regressores ou vetor de entrada do equalizador e $\boldsymbol{\Theta}(k)=$ $\left[\theta_{0} \theta_{1} \ldots, \theta_{M-1}\right] \in \mathbb{R}^{M \times 1}$ é o vetor de pesos do equalizador. $\mathrm{O}$ equalizador é modelado por uma estrutura descrita por um filtro adaptativo do tipo resposta finita impulsiva (FIR), em que o critério de adaptação é dado em função da minimização do erro quadrático de equalização $e(k)=d(k-L)-\hat{d}(k)=$ $d(k-L)-\boldsymbol{\Theta}^{T}(k) \mathcal{X}(k)$, em que $d(k)$ é o sinal de entrada e $L \in \mathbb{N}$ é o atraso de equalização.

\section{Algoritmo FVSS-NLMS}

De acordo com o critério de Wiener, a atualização da estimativa do vetor de pesos de um filtro adaptativo é obtida da seguinte forma:

$$
\boldsymbol{\Theta}(k+1)=\boldsymbol{\Theta}(k)-\frac{1}{2} \mu \nabla_{\boldsymbol{\Theta}(k)}\left(\mathrm{E}\left[e^{2}(k)\right]\right),
$$

em que $J=\nabla_{\boldsymbol{\Theta}(k)}\left(\mathrm{E}\left[e^{2}(k)\right]\right)$ é o funcional de custo descrito pelo gradiente estocástico do erro quadrático $e^{2}(k)=(d(k-$ $L)-\hat{d}(k))^{2}$ com $\hat{d}(k)=\boldsymbol{\Theta}^{T}(k) \mathcal{X}(k)$ e $\mu$ é o tamanho de passo de atualização da estimativa do vetor de pesos $\Theta(k)$. O objetivo do critério de Wiener é a minimização do gradiente estocástico $\nabla_{\boldsymbol{\Theta}(k)}\left(\mathrm{E}\left[e^{2}(k)\right]\right)$, em que a solução ótima é dada por $\boldsymbol{\Theta}_{0}=\mathbf{R}^{-1} \mathbf{p} \operatorname{com} \nabla_{\boldsymbol{\Theta}(k)}\left(\mathrm{E}\left[e^{2}(k)\right]\right)=2 \mathbf{R} \boldsymbol{\Theta}(k)-2 \mathbf{p}$, em que $\mathbf{R}=\mathrm{E}\left[\boldsymbol{\mathcal { X }}(k) \mathcal{X}^{T}(k)\right]$ é a matriz de autocorrelação e $\mathbf{p}=\mathrm{E}[\boldsymbol{\mathcal { X }}(k) d(k)]$ é o vetor de correlação cruzada.

Em um contexto de operação em tempo real é bastante dispendioso e inviável a acumulação de amostras para obtenção da matriz de autocorrelação e do vetor de correlação cruzada. No lugar de trabalhar com a esperança matemática $\mathrm{E}[\bullet]$ do erro quadrático, é possível trabalhar com valores instantâneos do erro quadrático. Sendo assim, o funcional de custo $J$ é reescrito da seguinte forma:

$$
J=\nabla_{\boldsymbol{\Theta}(k)}\left(e^{2}(k)\right)
$$

substituindo (4) em (3), obtém-se que:

$$
\begin{aligned}
\boldsymbol{\Theta}(k+1) & =\boldsymbol{\Theta}(k)-\frac{1}{2} \mu \nabla_{\boldsymbol{\Theta}(k)}\left(e^{2}(k)\right) \\
& =\boldsymbol{\Theta}(k)+\mu e(k) \mathcal{X}(k)
\end{aligned}
$$

em que $\nabla_{\boldsymbol{\Theta}(k)}\left(e^{2}(k)\right)=-2 e(k) \mathcal{X}(k)$. O algoritmo adaptativo NLMS é obtido através da normalização do algoritmo adaptativo LMS (5) pela potência do sinal de entrada, dado conforme a seguir:

$$
\boldsymbol{\Theta}(k+1)=\boldsymbol{\Theta}(k)+\mu \frac{e(k) \mathcal{X}(k)}{\mathcal{X}^{T}(k) \mathcal{X}(k)}
$$

Em (6), observa-se que o tamanho de passo é um parâmetro importante para atualização da estimativa do vetor de pesos do algoritmo NLMS, de tal forma que a velocidade de convergência, MSE e estabilidade do algoritmo são influenciados pelo valor do tamanho de passo. Na Seção II foi apresentado a declaração do problema de equalização adaptativa de canal de comunicação. Para que o equalizador possa reconstruir o sinal transmitido pelo canal de comunicação, é necessário que a estimativa do vetor de pesos $\boldsymbol{\Theta}(k)$ seja atualizada a cada instante. Nesse trabalho, a atualização da estimativa do vetor 
de pesos $\Theta(k)$ é realizada pelo algoritmo FVSS-NLMS, como segue:

$$
\begin{aligned}
& \text { FVSS-NLMS } \\
& \boldsymbol{\Theta}(k+1)= \begin{cases}\boldsymbol{\Theta}(k)+\mu(k) \frac{e(k) \mathcal{X}(k)}{g(k)}, & \text { se } g(k) \neq 0 \\
\boldsymbol{\Theta}(k), & \text { se } g(k)=0,\end{cases}
\end{aligned}
$$$$
k \in[1, K]
$$

em que $\mu(k)$ é o tamanho de passo adaptado pelo MFIS, $g(k)=\mathcal{X}^{T}(k) \mathcal{X}(k), \boldsymbol{\Theta}(k)$ é vetor de pesos do equalizador, $e^{2}(k)$ é o erro quadrático, $\mathcal{X}(k)$ é o vetor de regressores, $\mathcal{K}(k)$ é o instante de tempo normalizado pelo método Min-Max e $K$ é o número total de instantes de tempo.

Definição 1 (Função de Pertinência). Seja $U$ um universo de discurso e $x \in U$. Um conjunto fuzzy $F$ de $U$ é caracterizado por uma função:

$$
m_{F}(x): U \rightarrow[0,1]
$$

que é chamada de função de pertinência (do inglês, $M B F$ - Membership Function) do conjunto fuzzy F. A MBF $m_{F}$ associa $x$ ao conjunto fuzzy $F$ com um determinado grau de pertinência pertencente ao intervalo $[0,1]$.

Definição 2 (Variável Linguística). Seja uma variável linguística $x$ definida no universo de discurso U. Assim como uma variável numérica recebe valores numéricos, uma variável linguística recebe valores linguísticos. Por exemplo, $x$ é uma variável linguística definida como a "altura" que pode receber os valores linguísticos "baixa”, "média" ou "alta".

Definição 3 (Regra Fuzzy). Para um MFIS, as regras fuzzy do tipo Se (proposições do antecedente) então (proposições do consequente) podem ser definidas por especialistas $e$ nelas está representado o conhecimento humano subjetivo. $O$ antecedente e consequente são definidos por proposições fuzzy do tipo $x$ é $A$, em que $x$ é uma variável linguística definida no universo $U$ e A é um valor linguístico representado pelo conjunto fuzzy $\tilde{A}$ definido no universo U. Proposições fuzzy podem ser conectadas formando proposições fuzzy compostas, por exemplo, através do conectivo lógico “ $\boldsymbol{E}$ ”, que representa a interseção fuzzy caracterizada pela seguinte $M B F$ :

$$
m(x, y)=t\left[m_{\tilde{A}}(x), m_{\tilde{B}}(y)\right]=\min \left[m_{\tilde{A}}(x), m_{\tilde{B}}(y)\right],
$$

em que y é uma variável linguística definida no universo $V e$ $B$ é um valor linguístico representado pelo conjunto fuzzy $\tilde{B}$ definido no universo $V$.

O MFIS é composto por duas variáveis de entrada que são o erro quadrático $e^{2}(k)$ e $\mathcal{K}(k)$ que é o instante de tempo $k$ normalizado pelo método Min-Max. Cada variável de entrada que é definida como uma variável linguística do antecedente do MFIS, através da fuzzificação realizada a cada instante de tempo pela $j$-ésima $\mathrm{MBF}$, recebe cada valor linguístico do antecedente com um certo grau de pertinência através dos seguintes mapeamentos $m_{j}(\mathcal{K}(k)): U \rightarrow[0,1]$ e $m_{j}\left(e^{2}(k)\right): V \rightarrow[0,1]$, com os universos $U=[0,1]$ e $V=\left[0,1 \times 10^{-5}, 3 \times 10^{-5}\right]$. Foram definidas três
MBFs do tipo triangular para cada variável linguística do antecedente, com os valores linguísticos "Pequeno" (P) para $j=1$, "Médio" (M) para $j=2$ e "Grande" (G) para $j=3$, representados, respectivamente, pelo vetor de MBFs $\left[m_{1}\left(e^{2}(k)\right), m_{2}\left(e^{2}(k)\right), m_{3}\left(e^{2}(k)\right]\right.$ para a variável linguística $e^{2}(k)$ e pelo vetor de MBFs $\left[m_{1}(\mathcal{K}(k)), m_{2}(\mathcal{K}(k)), m_{3}(\mathcal{K}(k)]\right.$ para a variável linguística $\mathcal{K}(k)$. Na Tabela I são apresentados os parâmetros que definem as três MBFs do tipo triangular para os valores linguísticos do antecedente.

Tabela I: Intervalo das MBFs triangulares.

\begin{tabular}{c|c|c|c|c|c}
\hline \hline \multicolumn{2}{c|}{$\mathcal{K}(k)$} & \multicolumn{3}{c|}{$e^{2}(k)$} & \multicolumn{2}{c}{$\lambda(k)$} \\
\hline \multicolumn{2}{|c|}{ Intervalo } & \multicolumn{3}{c}{ Intervalo } & \multicolumn{2}{c}{ Intervalo } \\
\hline $\mathrm{P}$ & {$\left[\begin{array}{llll}0 & 0,2 & 0,3\end{array}\right]$} & $\mathrm{P}$ & {$\left[\begin{array}{llll}0,1 & 0,3 & 0,9\end{array}\right] \times 10^{-5}$} & $\mathrm{P}$ & {$\left[\begin{array}{llll}0,001 & 0,005 & 0,015\end{array}\right]$} \\
\hline $\mathrm{M}$ & {$\left[\begin{array}{llll}0,2 & 0,3 & 0,5\end{array}\right]$} & $\mathrm{M}$ & {$\left[\begin{array}{llll}0,3 & 0,9 & 1\end{array}\right] \times 10^{-5}$} & $\mathrm{M}$ & {$\left[\begin{array}{llll}0,005 & 0,015 & 0,03\end{array}\right]$} \\
\hline $\mathrm{G}$ & {$\left[\begin{array}{llll}0,3 & 0,5 & 1,0\end{array}\right]$} & $\mathrm{G}$ & {$\left[\begin{array}{lll}0,9 & 1 & 3\end{array}\right] \times 10^{-5}$} & $\mathrm{G}$ & {$\left[\begin{array}{lll}0,015 & 0,03 & 0,1\end{array}\right]$} \\
\hline \hline
\end{tabular}

A cada instante de tempo, a variável linguística do consequente $\lambda(k)$ recebe cada valor linguístico do consequente com um certo grau de pertinência pertencente ao intervalo $[0,1]$ através do seguinte mapeamento $m_{j}(\lambda(k)): Z \rightarrow[0,1]$, com o universo $Z=\left[1 \times 10^{-3}, 1 \times 10^{-1}\right]$. Foram definidas três MBFs do tipo triangular para a variável linguística do consequente, com os valores linguísticos "Pequeno" (P) para $j=1$, "Médio" (M) para $j=2$ e "Grande" (G) para $j=3$, representados, respectivamente, pelo vetor de MBFs $\left[m_{1}(\lambda(k)), m_{2}(\lambda(k)), m_{3}(\lambda(k))\right]$. Na Tabela I são apresentados os parâmetros que definem as três MBFs do tipo triangular para os valores linguísticos do consequente.

As MBFs do antecedente e consequente são definidas em função do conhecimento do especialista através de experiências passadas durante as simulações do problema analisado. A base de regras desenvolvida para adaptação paramétrica fuzzy do tamanho de passo $\mu(k)$ pode ser vista em (14). As proposições fuzzy do antecedente e consequente se relacionam através de uma proposição fuzzy condicional, tal que proposições do antecedente $\rightarrow$ proposição do consequente. A proposição fuzzy condicional é modelada pela relação fuzzy entre os universos de discurso $U \times V$ do antecedente e $Z$ do consequente, dada pela implicação fuzzy do antecedente para o consequente. A entrada da implicação fuzzy é o grau de ativação da $i$-ésima regra dado pela norma-t das MBFs do antecedente, conforme a seguir:

$$
\alpha^{i}=m\left(\mathcal{K}(k), e^{2}(k)\right)=\min \left[m_{j}(\mathcal{K}(k)), m_{j}\left(e^{2}(k)\right)\right],
$$

e a saída da implicação fuzzy é uma MBF que é obtida através do método do corte- $\alpha^{i}$, dado por:

$$
m_{\mathcal{R}^{i}}=\min \left[\alpha^{i}, m_{j}(\lambda(k))\right]
$$

Uma vez que a implicação fuzzy é realizada para a $i$-ésima regra, então uma MBF $m_{\mathcal{R}^{i}}$ é obtida para cada regra fuzzy. Para se obter uma única MBF que represente a resposta total do MFIS, é realizada a agregação fuzzy. Todos as MBFs $m_{\mathcal{R}^{i}}$ são combinadas através da agregação fuzzy, conforme a seguir:

$$
m_{\text {Total }}=\max \left[m_{\mathcal{R}^{1}}, m_{\mathcal{R}^{2}}, \ldots, m_{\mathcal{R}^{9}}\right]
$$


Após realizada a agregação fuzzy, é necessário obter um valor numérico para o tamanho de passo $\mu(k)$ através da defuzzificação da MBF $m_{\text {Total }}$. Para o algoritmo de otimização proposto, o método de defuzzificação utilizado é do tipo centroide, cujo valor numérico obtido é igual ao centro de área de $m_{\text {Total }}$, dado por:

$$
\mu(k)=\frac{\sum_{i=1}^{9} \lambda^{i}(k) m_{\text {Total }}\left(\lambda^{i}(k)\right)}{\sum_{i=1}^{9} m_{\text {Total }}\left(\lambda^{i}(k)\right)}
$$

$$
\begin{aligned}
& \mathcal{R}^{1}: \mathbf{S e} \mathcal{K}(k) \text { é } \mathbf{P} \mathbf{E} e^{2}(k) \text { é } \mathbf{P} \text { então } \lambda(k) \text { é } \mathbf{M} \\
& \mathcal{R}^{2}: \text { Se } \mathcal{K}(k) \text { é } \mathbf{P} \mathbf{E} e^{2}(k) \text { é } \mathbf{M} \text { então } \lambda(k) \text { é } \mathbf{M} \\
& \mathcal{R}^{3} \text { : Se } \mathcal{K}(k) \text { é } \mathbf{P} \mathbf{E} e^{2}(k) \text { é } \mathbf{G} \text { então } \lambda(k) \text { é } \mathbf{M} \\
& \mathcal{R}^{4}: \text { Se } \mathcal{K}(k) \text { é } \mathbf{M} \mathbf{E} e^{2}(k) \text { é } \mathbf{P} \text { então } \lambda(k) e ́ \text { é } \\
& \mathcal{R}^{5}: \text { Se } \mathcal{K}(k) \text { é } \mathbf{M} \mathbf{E} e^{2}(k) \text { é } \mathbf{M} \text { então } \lambda(k) \text { é } \mathrm{P} \\
& \mathcal{R}^{6} \text { : Se } \mathcal{K}(k) \text { é } \mathbf{M} \mathbf{E} e^{2}(k) \text { é } \mathbf{G} \text { então } \lambda(k) \text { é } \mathbf{G} \\
& \mathcal{R}^{7}: \text { Se } \mathcal{K}(k) \text { é } \mathbf{G} \mathbf{E} e^{2}(k) \text { é } \mathbf{P} \text { então } \lambda(k) \text { é } \mathbf{P} \\
& \mathcal{R}^{8}: \text { Se } \mathcal{K}(k) \text { é G E } e^{2}(k) \text { é M então } \lambda(k) \text { é } \mathbf{M} \\
& \mathcal{R}^{9}: \text { Se } \mathcal{K}(k) e ́ \text { G } \mathbf{E} e^{2}(k) \text { é } \mathbf{G} \text { então } \lambda(k) \text { é } \mathbf{G}
\end{aligned}
$$

\section{Simulação e Resultados}

\section{A. Simulação}

O sinal de entrada $d(k)$ é um sinal aleatório modulado via BPSK (do inglês, Binary Phase Shift Keying) resultando em um sinal codificado $a(k)$ descrito por uma sequência binária $\{ \pm 1\}$. O ruído aditivo $v(k)$ é uniformemente distribuído com relações sinal-ruído iguais a $\mathrm{SNR}=18 \mathrm{~dB}, \mathrm{SNR}=24 \mathrm{~dB}$ e SNR $=30 \mathrm{~dB}$. A resposta impulsiva do canal é dada por $h=\left[\begin{array}{llll}0,9 & 0,3 & 0,5 & -0,1\end{array}\right]$. O vetor de pesos $\boldsymbol{\Theta}(k)$ do equalizador foi definido com a quantidade de pesos igual a $M=10$. O número de amostras ou instantes de tempo do sinal de entrada foi definida com $K=4000$ e foram realizadas 100 simulações independentes de Monte Carlo. Os resultados obtidos através da equalização adaptativa via algoritmo FVSSNLMS foram comparados com o NLMS com o tamanho de passo fuzzy (versão do NMLS com o VSS adaptado apenas em função do erro quadrático por um MFIS proposto em [13]) e pelas versões tradicionais dos algoritmos LMS e NLMS com o tamanho de passo fixo. O tamanho de passo para os algoritmos LMS e NLMS foi definido com $\mu=0,03$.

\section{B. Resultados}

Nesta seção são apresentados os resultados obtidos através da equalização adaptativa do canal de comunicação apresentado na Seção IV-A. Nas Figuras 1, 5 e 9 são apresentadas as evoluções temporais do MSE para os algoritmos LMS, NLMS, NLMS com o tamanho de passo fuzzy [13] e FVSSNLMS, com as relações sinal-ruído iguais a $\mathrm{SNR}=18 \mathrm{~dB}$, $\mathrm{SNR}=24 \mathrm{~dB}$ e SNR $=30 \mathrm{~dB}$, respectivamente. Conforme pode ser visto nessas figuras, o algoritmo FVSS-NLMS obteve as melhores desempenhos em velocidade de convergência e em MSE no regime estacionário. A evolução temporal da adaptação do VSS é mostrada nas Figuras 4, 8 e 12, respectivamente, para as relações sinal-ruído $\mathrm{SNR}=18 \mathrm{~dB}$, $\mathrm{SNR}=24 \mathrm{~dB}$ e SNR $=30 \mathrm{~dB}$.
Quando comparado ao NMLS com tamanho de passo fuzzy [13], é possível notar o desempenho superior do algoritmo proposto nesse trabalho. O bom desempenho do algoritmo FVSS-NLMS é devido ao VSS ser adaptado através de uma descrição linguística baseado no conhecimento do especialista e implementada em uma base de regras fuzzy, cuja as entradas do MFIS são o erro quadrático e o instante de tempo normalizado pelo método Min-Max. Dessa forma, o VSS é adaptado em função do erro quadrático de equalização e do avanço temporal do processo de otimização realizado pelo algoritmo FVSS-NLMS. É possível notar que quanto menor a relação sinal-ruído pior é o desempenho do equalizador adaptativo com relação ao MSE no regime estacionário obtido; portanto os melhores resultados foram obtidos quando $\mathrm{SNR}=30 \mathrm{~dB}$ e os piores resultados quando $\mathrm{SNR}=18 \mathrm{~dB}$.

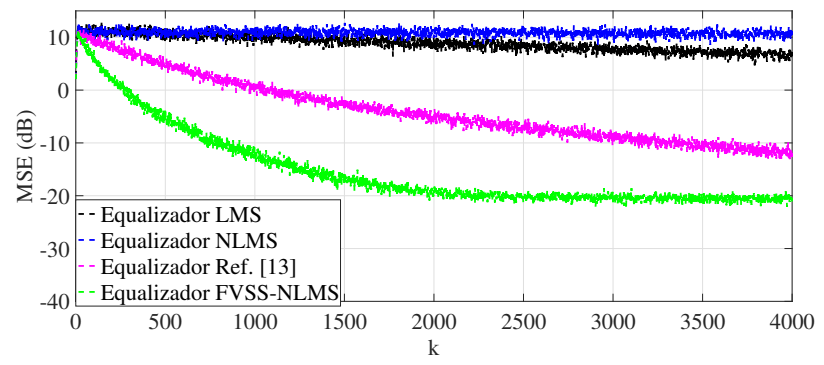

Figura 1: MSE para SNR $=30 \mathrm{~dB}$.

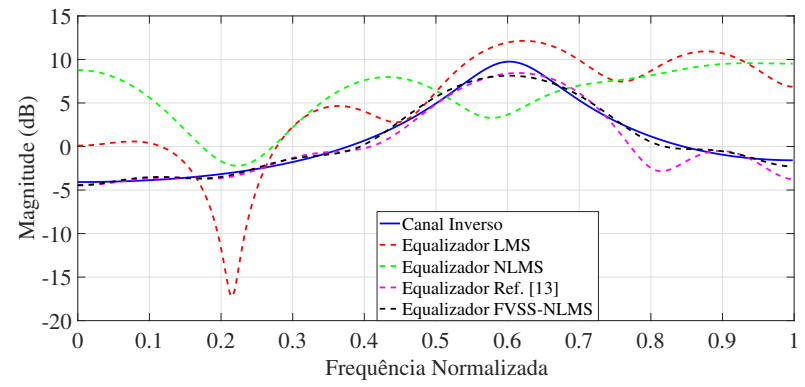

Figura 2: Resposta em magnitude do canal equalizado para $\mathrm{SNR}=30 \mathrm{~dB}$.

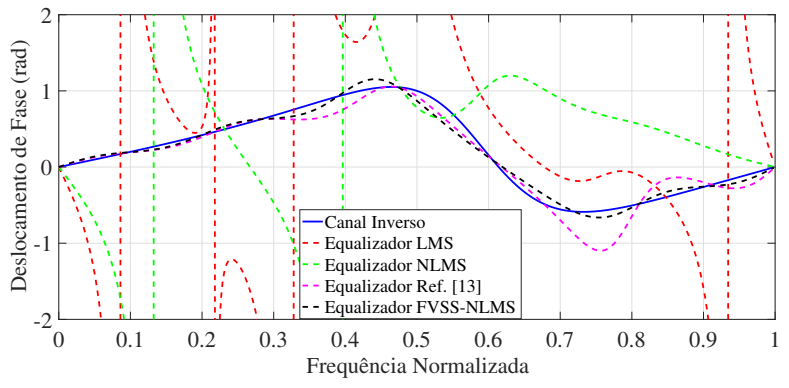

Figura 3: Resposta em fase do canal equalizado para SNR = $30 \mathrm{~dB}$. 


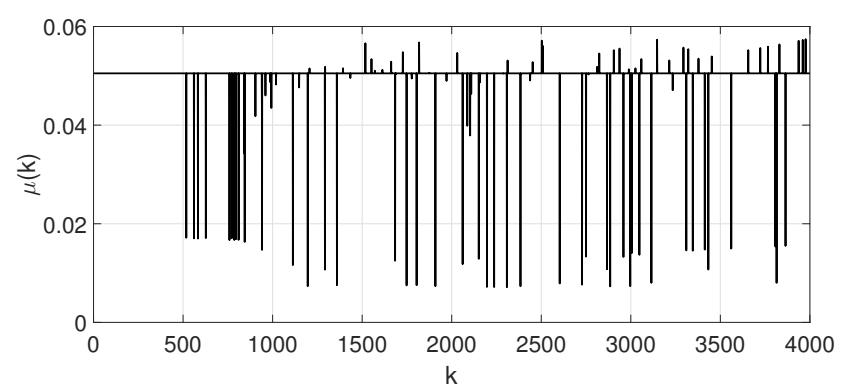

Figura 4: Tamanho de passo variável para $\mathrm{SNR}=30 \mathrm{~dB}$.

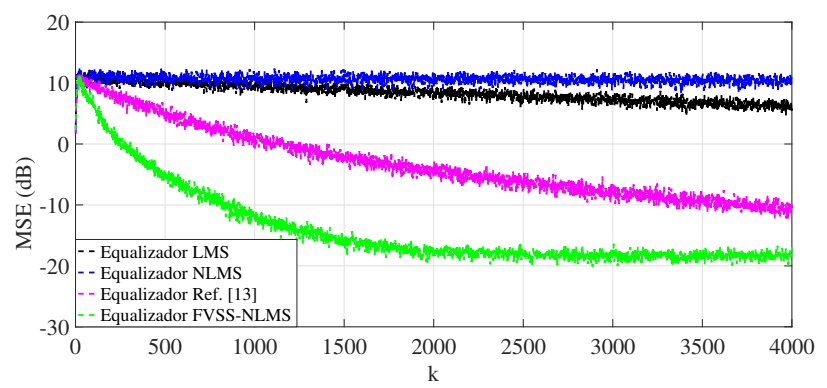

Figura 5: MSE para SNR $=24 \mathrm{~dB}$.

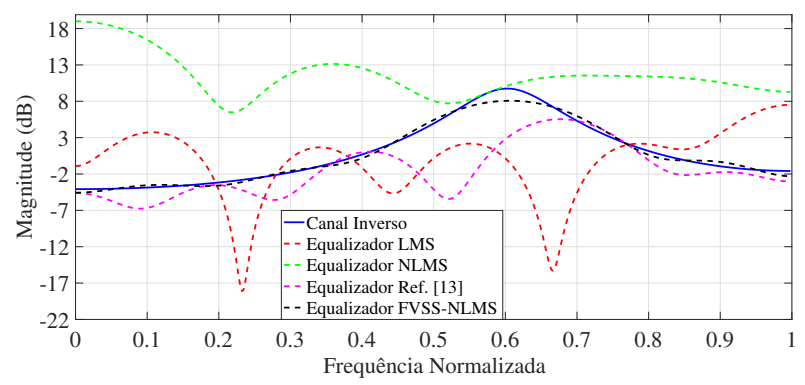

Figura 6: Resposta em magnitude do canal equalizado para $\mathrm{SNR}=24 \mathrm{~dB}$.

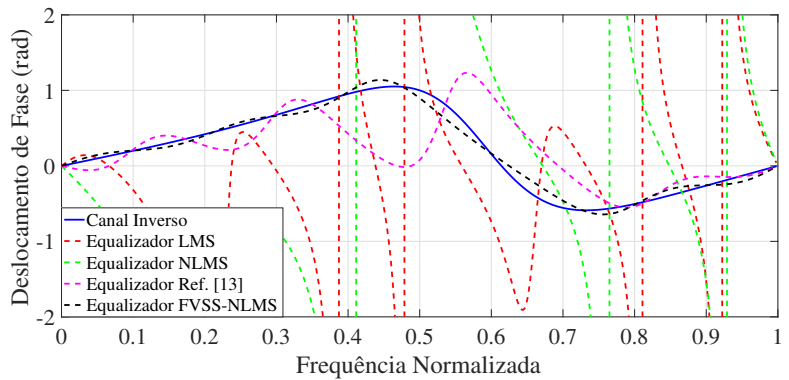

Figura 7: Resposta em fase do canal equalizado para SNR = $24 \mathrm{~dB}$.

Para verificar se a equalização adaptativa do canal foi realizada de forma satisfatória, os resultados obtidos nas respostas em magnitude (Figuras 2, 6 e 10) e em fase (Figuras 3, 7 e 11) foram comparadas com os resultados obtidos pelo

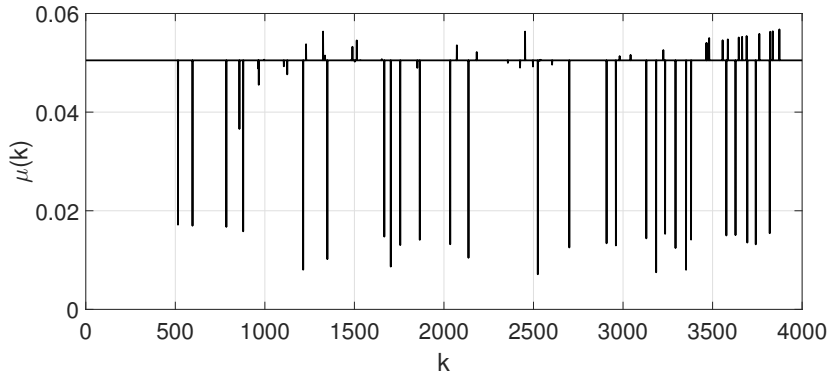

Figura 8: Tamanho de passo variável para $\mathrm{SNR}=24 \mathrm{~dB}$.

canal inverso. Dessa forma, as resposta em magnitude e em fase do canal inverso são as respostas de referência. Através dessas figuras, nota-se que o equalizador adaptativo projetado pelo algoritmo FVSS-NLMS obteve os melhores resultados em rastreabilidade da resposta em magnitude e em fase.

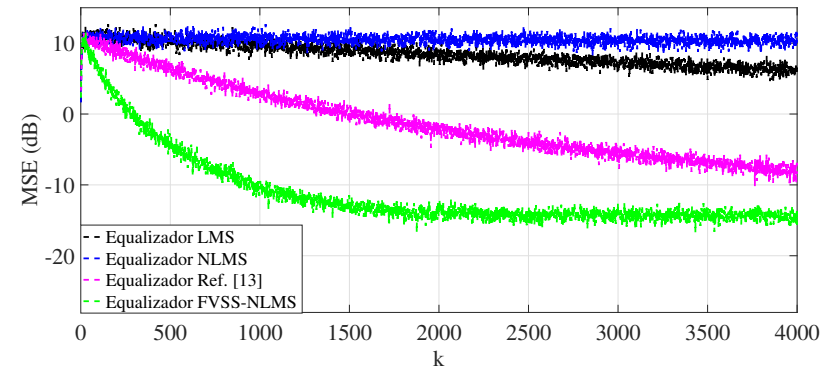

Figura 9: MSE para $\mathrm{SNR}=18 \mathrm{~dB}$.

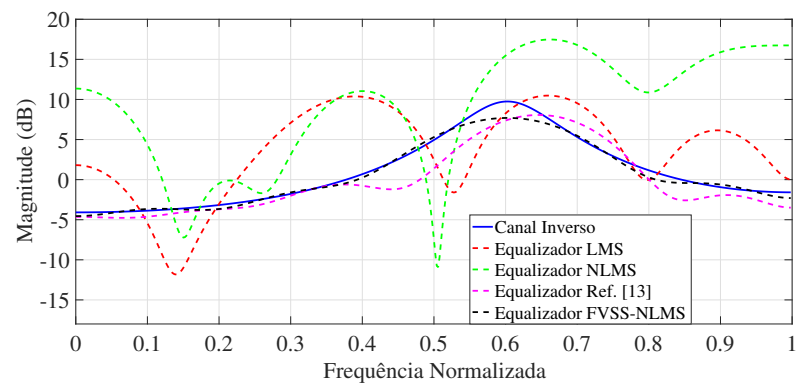

Figura 10: Resposta em magnitude do canal equalizado para $\mathrm{SNR}=18 \mathrm{~dB}$.

\section{CONCLUSÃO}

Nesse artigo, foi proposta a equalização adaptativa de canais lineares e invariantes no tempo através de uma nova versão do algoritmo NLMS com o VSS adaptado por um MFIS, intitulado FVSS-NLMS. O desempenho do equalizador adaptativo foi avaliado através de extensivas simulações e realizada a comparação com os resultados obtidos pelo NMLS com o tamanho de passo fuzzy [13] e pelas versões tradicionais dos algoritmos LMS e NLMS com o tamanho de passo fixo, para as relações sinal-ruído com $\mathrm{SNR}=18 \mathrm{~dB}, \mathrm{SNR}=24 \mathrm{~dB}$ e 


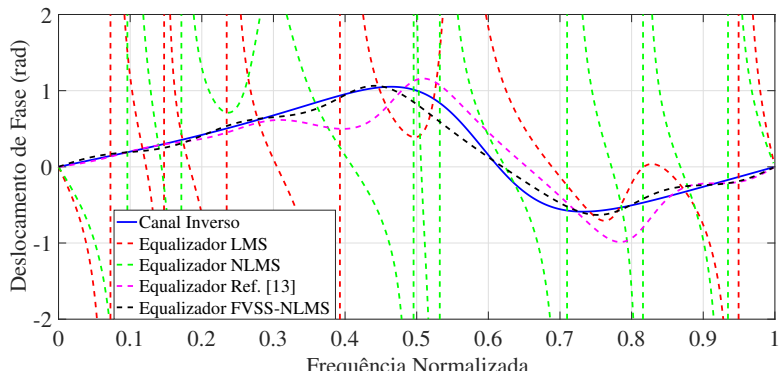

Figura 11: Resposta em fase do canal equalizado para SNR = $18 \mathrm{~dB}$.

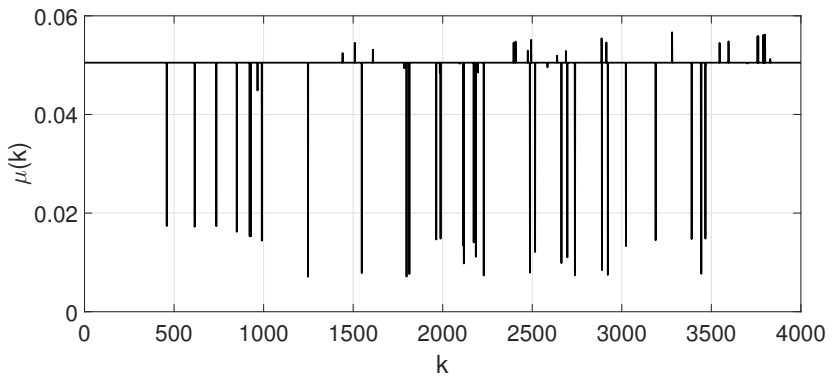

Figura 12: Tamanho de passo variável para $\mathrm{SNR}=18 \mathrm{~dB}$.

$\mathrm{SNR}=30 \mathrm{~dB}$. Conforme o esperado, através da adaptação do tamanho de passo realizada em função de uma descrição linguística baseada no conhecimento do especialista, foram obtidos resultados satisfatórios para algoritmo FVSS-NLMS, tanto em velocidade de convergência como em MSE no regime estacionário. Além disso, o desempenho do equalizador adaptativo foi comparado com as respostas em magnitude e em fase do canal inverso, em que foi observado o desempenho superior para o equalizador projetado pelo algoritmo FVSS-NLMS. Também pode ser notado que o algoritmo FVSS-NLMS é menos sensível à diminuição da relação sinal-ruído, em que para SNR $=18 \mathrm{~dB}$ satisfatórios resultados foram obtidos quando comparado aos resultados obtidos pelos algoritmos LMS e NLMS. Por fim, nota-se que a adaptação do VSS pelo MFIS independe de medidas estatísticas de alta ordem.

\section{REFERÊNCIAS}

[1] J. Li, C. Lim, and A. Nirmalathas, "Indoor optical wireless communications using few-mode based uniform beam shaping and $1 \mathrm{~ms}$ based adaptive equalization," in 2020 IEEE Photonics Conference (IPC). IEEE, 2020, pp. 1-2.

[2] E. Vlachos, A. S. Lalos, and K. Berberidis, "Stochastic gradient pursuit for adaptive equalization of sparse multipath channels," IEEE Journal on Emerging and Selected Topics in Circuits and Systems, vol. 2, no. 3, pp. 413-423, 2012.

[3] P. Zhu, X. Xu, X. Zhang, R. Wang, and X. Zhang, "A new variable step-size $1 \mathrm{~ms}$ algorithm for application to underwater acoustic channel equalization," in 2017 IEEE International Conference on Signal Processing, Communications and Computing (ICSPCC). IEEE, 2017, pp. $1-4$.

[4] S. Khan, A. Wahab, I. Naseem, and M. Moinuddin, "Comments on design of fractional-order variants of complex lms and nlms algorithms for adaptive channel equalization," Nonlinear Dynamics, vol. 101, no. 2, pp. 1053-1060, 2020
[5] A. A. Khan, S. M. Shah, M. A. Z. Raja, N. I. Chaudhary, Y. He, and J. T. Machado, "Fractional lms and nlms algorithms for line echo cancellation," Arabian Journal for Science and Engineering, pp. 1-14, 2021.

[6] A. Pandey, L. Malviya, and V. Sharma, "Comparative study of $1 \mathrm{~ms}$ and nlms algorithms in adaptive equalizer," International Journal of Engineering Research and Applications (IJERA), vol. 2, no. 3, pp. 15841587, 2012.

[7] M. S. Aslam, P. Shi, and C.-C. Lim, "Self-adapting variable step size strategies for active noise control systems with acoustic feedback," Automatica, vol. 123, p. 109354, 2021.

[8] T. Strutz, "Estimation of measurement-noise variance for variable-stepsize nlms filters," in 2019 27th European Signal Processing Conference (EUSIPCO). IEEE, 2019, pp. 1-5.

[9] F. Casco-Sanchez, M. Lopez-Guerrero, S. Javier-Alvarez, and R. C. Medina-Ramirez, "A variable-step size nlms algorithm based on the cross-correlation between the squared output error and the near-end input signal," IEEJ Transactions on Electrical and Electronic Engineering, vol. 14, no. 8, pp. 1197-1202, 2019.

[10] Q. Ren and P. Bigras, "A highly accurate model-free motion control system with a mamdani fuzzy feedback controller combined with a tsk fuzzy feed-forward controller," Journal of Intelligent \& Robotic Systems, vol. 86, no. 3-4, pp. 367-379, 2017.

[11] A. K. Sharma, D. Singh, and N. K. Verma, "Data driven aerodynamic modeling using mamdani fuzzy inference systems," in 2018 International Conference on Sensing, Diagnostics, Prognostics, and Control (SDPC). IEEE, 2018, pp. 359-364.

[12] A. Najmurrokhman, U. Komarudin, A. Sadiyoko, T. Y. Iskanto et al., "Mamdani based fuzzy logic controller for a wheeled mobile robot with obstacle avoidance capability," in 2019 International Conference on Mechatronics, Robotics and Systems Engineering (MoRSE). IEEE, 2019, pp. 49-53.

[13] Y. Ng, H. Mohamad, and T. Chuah, "Block-based fuzzy step size nlms algorithms for subband adaptive channel equalisation," IET Signal Processing, vol. 3, no. 1, pp. 23-32, 2009.

[14] J. M. T. Romano, R. Attux, C. C. Cavalcante, and R. Suyama, Unsupervised signal processing: channel equalization and source separation. CRC Press, 2018. 